\title{
Reduction of shunt dependency rates following aneurysmal subarachnoid hemorrhage by tandem fenestration of the lamina terminalis and membrane of Liliequist during microsurgical aneurysm repair
}

\author{
Ethan A. Winkler, MD, PhD, Jan-Karl Burkhardt, MD, W. Caleb Rutledge, MD, \\ Jonathan W. Rick, BS, Carlene P. Partow, BS, John K. Yue, BS, Harjus Birk, MD, \\ Ashley M. Bach, BA, Kunal P. Raygor, MD, and Michael T. Lawton, MD \\ Department of Neurological Surgery, University of California, San Francisco, California
}

\begin{abstract}
OBJECTIVE Shunt-dependent hydrocephalus is an important cause of morbidity following aneurysmal subarachnoid hemorrhage (aSAH) in excess of $20 \%$ of cases. Hydrocephalus leads to prolonged hospital and ICU stays, well as to repeated surgical interventions, readmissions, and complications associated with ventriculoperitoneal (VP) shunts, including shunt failure and infection. Whether variations in surgical technique at the time of aneurysm treatment may modify rates of shunt dependency remains a matter of debate. Here, the authors report on their experience with tandem fenestration of the lamina terminalis (LT) and membrane of Liliequist (MoL) at the time of open microsurgical repair of the ruptured aneurysm.
\end{abstract}

METHODS The authors conducted a retrospective review of 663 consecutive patients with aSAH treated from 2005 to 2015 by open microsurgery via a pterional or orbitozygomatic craniotomy by the senior author (M.T.L.). Data collected from review of the electronic medical record included age, Hunt and Hess grade, Fisher grade, need for an external ventricular drain, and opening pressure. Patients were stratified into those undergoing no fenestration and those undergoing tandem fenestration of the LT and MoL at the time of surgical repair. Outcome variables, including VP shunt placement and timing of shunt placement, were recorded and statistically analyzed.

RESULTS In total, shunt-dependent hydrocephalus was observed in $15.8 \%$ of patients undergoing open surgical repair following aSAH. Tandem microsurgical fenestration of the LT and MoL was associated with a statistically significant reduction in shunt dependency ( $17.9 \%$ vs $3.2 \%, p<0.01)$. This effect was confirmed with multivariate analysis of collected variables (multivariate $\mathrm{OR} 0.09,95 \% \mathrm{Cl} 0.03-0.30$ ). Number-needed-to-treat analysis demonstrated that tandem fenestration was required in approximately 6.8 patients to prevent a single VP shunt placement. A statistically significant prolongation in days to VP shunt surgery was also observed in patients treated with tandem fenestration $(26.6 \pm 19.4$ days vs $54.0 \pm 36.5$ days, $p<0.05$ ).

CONCLUSIONS Tandem fenestration of the $\mathrm{LT}$ and MoL at the time of open microsurgical clipping and/or bypass to secure ruptured anterior and posterior circulation aneurysms is associated with reductions in shunt-dependent hydrocephalus following aSAH. Future prospective randomized multicenter studies are needed to confirm this result.

https://thejns.org/doi/abs/10.3171/2017.5.JNS163271

KEY WORDS subarachnoid hemorrhage; hydrocephalus; aneurysm; ventriculoperitoneal shunt; vascular disorders

$\Lambda$ NEURYSMAL subarachnoid hemorrhage (aSAH) is a subtype of hemorrhagic stroke, with high neurological morbidity and mortality rates, that affects approximately 6-9 individuals per 100,000 people per year. ${ }^{7,36}$ In the United States alone, there are up to 30,000 new cases of aSAH per year. ${ }^{36}$ Improvements in multidis- ciplinary neurocritical care and advancements in medical and surgical treatment have contributed to a decline in the case fatality rate of aSAH. ${ }^{19}$ A greater proportion of patients, therefore, are surviving their initial hemorrhagic event but remain at increased risk of a number of complications, including delayed cerebral ischemia, epilepsy,

ABBREVIATIONS aSAH = aneurysmal subarachnoid hemorrhage; $E V D=$ external ventricular drain; $L T=$ lamina terminalis; $M o L=$ membrane of Liliequist; $V P=$ ventriculoperitoneal.

SUBMITTED December 31, 2016. ACCEPTED May 30, 2017.

INCLUDE WHEN CITING Published online December 15, 2017; DOI: 10.3171/2017.5.JNS163271. 
nosocomial infections, cognitive impairment, shunt-dependent hydrocephalus, and shunt-related complications. ${ }^{6}$

Hydrocephalus complicates the clinical course of greater than $20 \%$ of patients with aSAH..$^{32,33}$ Reported rates of hydrocephalus, however, are highly variable, with a reported range of $6.5 \%-67 \% .111,16,25,32,33$ Development of post-aSAH hydrocephalus is associated with increased neurological morbidity and prolongation of ICU and/or hospital stay. ${ }^{1,23,25,32,37}$ Hydrocephalus is often managed acutely with placement of an external ventricular drain (EVD). ${ }^{31}$ Failure to wean from drainage of CSF necessitates permanent CSF diversion-most commonly a ventriculoperitoneal (VP) shunt-in $8 \%-63 \%$ of patients. ${ }^{18}$

Shunt-dependent hydrocephalus is not without complications and leads to numerous undesirable sequelae, including shunt malfunction infections, overdrainage, and hospital readmissions. ${ }^{21,24}$ Reported revision rates are as high as $30 \%-47 \%$, and shunt malfunction remains the most common cause of hospital readmission following aSAH. ${ }^{10,22-24}$ Efforts have therefore been taken to identify modifiable risk factors and the optimal timing of intervention for shunt-dependent hydrocephalus following aSAH. ${ }^{1,22,27,32}$ Whether variation of surgical technique and alteration of CSF flow at the time of microsurgical treatment of the ruptured aneurysm reduce rates of shunt dependency following aSAH remains a matter of debate. ${ }^{13-15}$

Here, we report a surgical experience with tandem fenestration of the lamina terminalis (LT) and membrane of Liliequist (MoL) at the time of open microsurgical repair of the ruptured aneurysms. We demonstrate that tandem fenestration is associated with reduced rates of shunt-dependent hydrocephalus and may decrease the need for a VP shunt in patients following aSAH.

\section{Methods}

\section{Patient Population}

A retrospective, consecutive case series was collected of patients treated between January 2005 and December 2015. Inclusion criteria were as follows: adults (age $\geq 18$ years); SAH from an aneurysm, confirmed by preoperative CT and conventional angiography; and survival beyond initial hospitalization. Patients with ruptured aneurysms of the pericallosal artery, posterior inferior cerebellar artery, anterior inferior cerebellar artery, and vertebral artery were excluded from the present analysis because the surgical approach did not grant access to both the LT and MoL. All patients underwent microsurgical clip reconstruction and/or bypass performed by the senior author (M.T.L.) to secure the ruptured aneurysm and/or additional aneurysms identified at the time of the operation. ${ }^{17}$ Tandem fenestration of the LT and MoL was systematically performed in all patients with ruptured aneurysms starting in January 2014 onward and documented in the operative records. Approval from the University of California, San Francisco, Institutional Review Board was obtained before medical records were accessed.

\section{Surgical Technique}

Either a pterional or orbitozygomatic craniotomy, depending on aneurysm location and morphology, was per- formed in standard fashion, as previously described. ${ }^{17} \mathrm{Af}-$ ter opening the dura matter, subarachnoid dissection was begun and tandem fenestration was performed in typical fashion (Fig. 1). First, the sylvian fissure was widely split and the carotid cistern opened. If the aneurysm was not initially encountered-for example, in cases of an aneurysm of the carotid terminus or middle cerebral arterythe LT cistern was opened to mobilize the frontal lobe and the LT was widely fenestrated to release CSF and relax the brain. Dissection was next performed along the posterior communicating artery and anterior choroidal artery in the carotid-oculomotor triangle to open the crural cistern and detach the temporal lobe. Next, the MoL was widely opened medial to the oculomotor nerve, and the interpeduncular cistern was entered. This final maneuver brings the apex of the basilar artery and posterior cerebral artery into view. When possible, tandem fenestration was performed before treating the aneurysm to drain CSF, relax the brain, and optimize exposure. When the LT or MoL or both were inaccessible or tandem fenestration risked rerupturing the aneurysm, tandem fenestration was deferred until after clipping.

\section{Data Collection}

Patient demographic information (age and sex) and clinical characteristics (Hunt and Hess grade on admission and need for an EVD) were collected through retrospective review of the electronic medical record. Fisher grade and aneurysm location were assigned through careful review of the preoperative noncontrast CT scan and preoperative angiogram or CT angiogram, respectively. For the purposes of assigning aneurysm location, posterior communicating artery aneurysms were assigned a posterior location, as recently described, to ensure consistency with a recent meta-analysis identifying predictors of shunt-dependent hydrocephalus following aSAH. ${ }^{32}$ Similarly, high Hunt and Hess grade was defined as a grade of III to V and high Fisher score was defined as a score of 3 or 4 , as previously described..$^{32}$ Interval of follow-up was defined as the date of initial hospitalization to the last documented neurosurgical note.

\section{Statistical Analysis}

Descriptive statistics are presented as means and SDs for continuous variables and proportions for categorical variables. Differences in demographics and aSAH characteristics across intervention groups were assessed using Pearson's chi-square test for categorical variables, ANOVA for continuous variables, and the Mann-Whitney U-test for nonparametric variables. Fisher's exact test was used to assess for differences in categorical variables with individual cell counts $\leq 5$. For outcomes analysis, univariate logistic regression was performed with shunt placement (yes/no) as the outcome variable and demographic, clinical, and intervention variables as predictors. Multivariable logistic regression was performed to assess the independent association between the intervention group and other demographic and clinical predictors on shunt placement as the outcome. Odds ratios and their associated $95 \%$ confidence intervals are reported for each predic- 


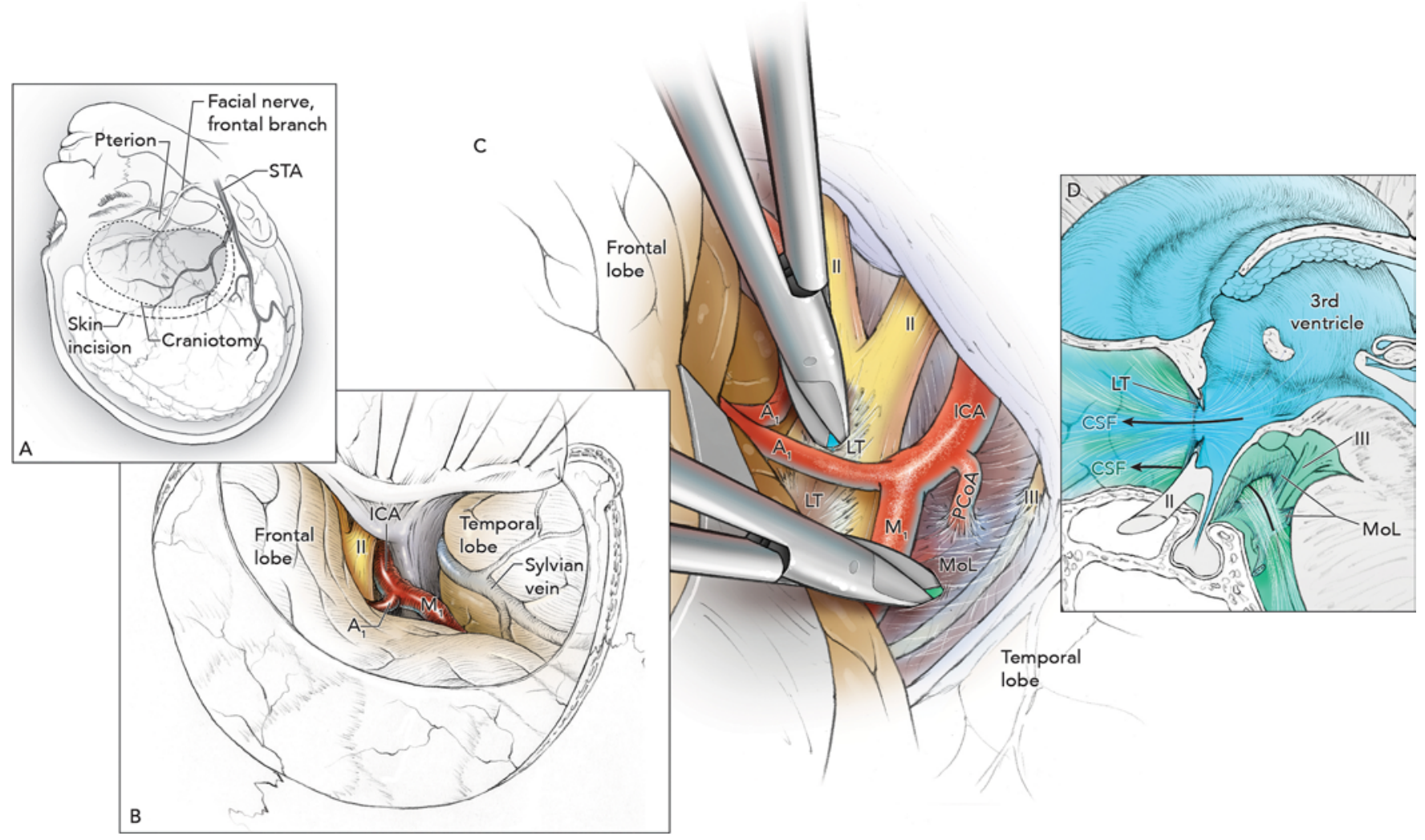

FIG. 1. Illustration depicting microsurgical technique for tandem fenestration of the LT and MoL to promote circulation of CSF following aSAH. A and B: The patient is positioned supine with head rotated to the contralateral side and extended to bring the malar prominence to the highest point of the operative field. A pterional or orbitozygomatic craniotomy is performed in standard fashion, and subarachnoid dissection is begun. C: After the sylvian fissure is split, the carotid and LT cisterns are widely opened, and the LT is widely fenestrated to release CSF and relax the brain. Subarachnoid dissection is then continued along the posterior communicating artery $(\mathrm{PCOA}$ ) and anterior choroidal artery in the carotid-oculomotor triangle. The MoL is then widely fenestrated medial to the oculomotor (III) nerve, and CSF is released from the interpeduncular cistern. D: Tandem fenestration of the LT and MoL allows communication between the infratentorial (green) and supratentorial (blue) compartments and unobstructed circulation of CSF to the absorptive arachnoid granulations over the convexity. Tandem fenestration promotes the clearance of subarachnoid blood products and prevents the development of hydrocephalus. $A_{1}=$ first segment of anterior cerebral artery; ICA = internal carotid artery; $I I=$ optic nerve; $\mathrm{M}_{1}$ = first segment of middle cerebral artery; STA = superficial temporal artery. Copyright University of California, San Francisco. Published with permission.

tor in the regression analyses. Significance was assessed at $\alpha=0.05$. All analyses were performed using IBM SPSS version 22 (IBM Corp.).

\section{Results}

\section{Patient Demographic and Clinical Variables}

From 2005 to 2015, 663 patients with aSAH underwent open surgical treatment of a ruptured aneurysm at the University of California, San Francisco, and met inclusion criteria. Overall, the mean follow-up duration was $305.0 \pm$ 26.2 days. Patient demographics and clinical information are summarized in Table 1. With respect to demographic and clinical characteristics, there were several noted differences between the nonfenestration and tandem fenestration groups. The tandem fenestration group was composed of patients with a statistically significantly greater age (mean age 58.3 vs 54.1 years, $\mathrm{p}<0.01$ ), higher Hunt and Hess grade on presentation (mean grade $2.8 \pm 1.2 \mathrm{vs}$ $2.5 \pm 1.1, \mathrm{p}<0.05$ ), higher Fisher grade (mean Fisher grade $3.4 \pm 0.9$ vs $3.1 \pm 1.1, \mathrm{p}<0.01)$, a greater proportion with intraventricular hemorrhage $(69.9 \%$ vs $56.4 \%, \mathrm{p}<0.05)$, and higher frequency of EVD insertions (percentage with EVD $52.7 \%$ vs $38.2 \%$, p < 0.01). No statistically significant intergroup differences were observed with age, sex, aneurysm location, or opening pressure with EVD placement. Prior to the systematic implementation of tandem fenestration, the MoL was only fenestrated when required to access a ruptured aneurysm in the posterior circulation, such as aneurysms of the basilar apex, posterior cerebral artery, or superior cerebellar artery. Fenestration of the MoL was therefore only performed in isolation in 64 (11.2\%) of 570 cases in the nonfenestration group.

\section{Tandem Fenestration Is Associated With Reduced Post-aSAH Shunt-Dependent Hydrocephalus}

There were no complications specifically related to fenestrating the LT or MoL. Overall, placement of a VP shunt for permanent CSF diversion was required in 105 patients with aSAH (15.8\%). Only $9(8.6 \%)$ and $2(1.2 \%)$ of the 105 patients required a VP shunt at an interval greater 
TABLE 1. Demographic and clinical characteristics of patients with aSAH stratified by treatment

\begin{tabular}{|c|c|c|c|c|}
\hline \multirow[b]{2}{*}{ Variable } & \multirow{2}{*}{$\begin{array}{l}\text { Overall Pop } \\
\qquad(n=663)\end{array}$} & \multicolumn{2}{|c|}{ Treatment Group } & \multirow[b]{2}{*}{$\mathrm{p}$ Value } \\
\hline & & Nonfenestration $(n=570)$ & Tandem Fenestration $(n=93)$ & \\
\hline Age (yrs) & & & & 0.001 \\
\hline Mean & $54.5 \pm 14.2$ & $53.8 \pm 14.2$ & $59.1 \pm 13.8$ & \\
\hline Sex & & & & 0.430 \\
\hline Male & $198(29.9)$ & $167(29.3)$ & $31(33.3)$ & \\
\hline Female & $465(70.1)$ & $403(70.7)$ & $62(66.7)$ & \\
\hline Aneurysm location & & & & 0.571 \\
\hline Anterior & $461(69.5)$ & $394(69.1)$ & $67(72.0)$ & \\
\hline Posterior & $202(30.5)$ & $176(30.9)$ & $26(28.0)$ & \\
\hline \multicolumn{5}{|l|}{ Hunt \& Hess score } \\
\hline Low (1-2) & $396(59.7)$ & $353(61.9)$ & $43(46.2)$ & \\
\hline High (3-5) & $267(40.3)$ & $217(38.1)$ & $50(53.8)$ & \\
\hline Mean & $2.53 \pm 1.08$ & $2.49 \pm 1.06$ & $2.81 \pm 1.19$ & 0.008 \\
\hline Fisher grade & & & & 0.048 \\
\hline Low (1-2) & $153(23.1)$ & $139(24.4)$ & $14(15.1)$ & \\
\hline High (3-4) & $510(76.9)$ & $431(75.6)$ & $79(84.9)$ & \\
\hline Mean & $3.12 \pm 1.07$ & $3.07 \pm 1.10$ & $3.40 \pm 0.85$ & 0.006 \\
\hline Intraventricular hemorrhage & & & & 0.015 \\
\hline No & $276(41.6)$ & $248(43.5)$ & $28(30.1)$ & \\
\hline Yes & $387(58.4)$ & $322(56.5)$ & $65(69.9)$ & \\
\hline EVD placement & & & & 0.008 \\
\hline No & $396(59.7)$ & $352(61.8)$ & $44(47.3)$ & \\
\hline Yes & $267(40.3)$ & $218(8.2)$ & $49(52.7)$ & \\
\hline \multicolumn{5}{|l|}{ Opening pressure } \\
\hline Mean & $17.4 \pm 10.1$ & $17.4 \pm 10.5$ & $17.4 \pm 9.2$ & 0.979 \\
\hline
\end{tabular}

Pop = population.

Values expressed as the mean \pm SD or as number (\%) unless otherwise indicated.

than 2 months and 1 year after initial hospitalization, respectively. Tandem fenestration of the LT and MoL was associated with a statistically significant $82 \%$ reduction in the incidence of shunt-dependent hydrocephalus (percentage requiring VP shunt $3.2 \%$ vs $17.9 \%$, p < 0.01). Tandem fenestration was also associated with a statistically significant 27.8-day delay in the timing of shunt insertion (mean days to VP shunt placement $54.0 \pm 36.5$ vs $26.6 \pm$ 19.4) (Table 2). Univariate statistical analysis confirmed that tandem fenestration was associated with a statistically significantly lower odds of VP shunt placement (univariate OR 0.15, 95\% CI 0.05-0.49). High Hunt and Hess grade, high Fisher score, the presence of intraventricular blood, posterior aneurysm location, and the insertion of an EVD were conversely associated with a greater univariate odds ratio of requiring VP shunt placement. No relationship was observed between age and/or sex and odds of need for a VP shunt following aSAH (Table 3).

To minimize potential confounding of baseline differences of demographic and clinical variables between tandem fenestration and nonfenestration cohorts, we next proceeded with multivariate analyses of all studied variables. The association between tandem fenestration of the LT and MoL with statistically significantly lower odds of

TABLE 2. Rates of shunt-dependent hydrocephalus and timing of VP shunt placement in patients with aSAH stratified by treatment

\begin{tabular}{lcccc}
\hline & & \multicolumn{2}{c}{ Treatment Group } & \\
\cline { 3 - 4 } Variable & Overall & Nonfenestration & Tandem Fenestration & $p$ Value \\
\hline Shunt dependency & & & & 0.002 \\
\hline No & $558(84.2)$ & $468(82.1)$ & $90(96.8)$ & \\
\hline Yes & $105(15.8)$ & $102(17.9)$ & $3(3.2)$ & \\
\hline Mean days to VP shunt placement & $27.4 \pm 20.4$ & $26.6 \pm 19.4$ & $54.0 \pm 36.5$ & 0.021 \\
\hline
\end{tabular}

Values expressed as the mean \pm SD or as number (\%) unless otherwise indicated. 
TABLE 3. Univariate and multivariate odds ratios of clinical predictors of shunt-dependent hydrocephalus following aSAH

\begin{tabular}{lcccc}
\hline \multicolumn{1}{c}{ Predictor } & Univariate OR $(95 \% \mathrm{Cl})$ & $\mathrm{p}$ Value & Multivariate OR $(95 \% \mathrm{Cl})$ & $\mathrm{p} \mathrm{Value}$ \\
\hline Age, per yr & $1.01(1.00-1.03)$ & 0.12 & $1.01(0.99-1.03)$ & 0.31 \\
\hline Female sex & $1.21(0.75-1.93)$ & 0.44 & $1.11(0.66-1.86)$ & 0.71 \\
\hline Posterior circulation aneurysm & $1.74(1.13-2.68)$ & 0.01 & $1.60(1.00-2.57)$ & 0.05 \\
\hline High Hunt \& Hess grade & $3.16(2.05-4.87)$ & $<0.001$ & $1.68(0.99-2.84)$ & 0.05 \\
\hline High Fisher grade & $2.62(1.40-4.92)$ & 0.003 & $1.28(0.62-2.64)$ & 0.50 \\
\hline Intraventricular hemorrhage & $2.47(1.54-3.96)$ & $<0.001$ & $0.68(0.39-1.14)$ & $<.67(2.13-6.32)$ \\
\hline EVD placement & $5.04(3.17-7.99)$ & $<0.001$ & $0.09(0.03-0.30)$ & $<0.001$ \\
\hline Tandem fenestration of LT \& MoL & $0.15(0.05-0.49)$ & 0.002 & &
\end{tabular}

VP shunt insertion withstood multivariate analysis (multivariate OR $0.09,95 \%$ CI $0.03-0.30$ ). This analysis similarly confirmed a statistically significant 3.67 -fold greater odds of post-aSAH VP shunt placement in patients requiring EVD insertion for acute hydrocephalus. Our multivariable model failed to show a statistically greater odds of shunt-dependent hydrocephalus with age, sex, posterior aneurysm location, high Hunt and Hess grade, high Fisher grade, or the presence of intraventricular hemorrhage (Table 3).

\section{Number-Needed-to-Treat Analysis}

Having demonstrated a reduction in post-aSAH shunt dependency with tandem fenestration, we estimated effect size of this surgical technique. The absolute risk reduction of shunt dependency with tandem fenestration was $14.7 \%$. Therefore, 6.8 patients would have to undergo tandem fenestration of the LT and MoL to prevent a single VP shunt placement following aSAH. Prior works, as well as our own univariable analysis, demonstrated greater risk in those with greater subarachnoid blood, as evidenced by a Fisher grade of 3 or 4 , the presence of intraventricular hemorrhage, and/or need for placement of an EVD. ${ }^{1,32} \mathrm{In}$ subgroup analysis of those at greatest risk, we found an even lower number needed to treat in individuals at greatest risk for shunt-dependent hydrocephalus, as evidence by a number needed to treat of 4.8, 5.8, and 3.7 in those with intraventricular hemorrhage, a high Fisher grade, and requiring placement of an EVD, respectively.

\section{Discussion}

The etiology of hydrocephalus following aSAH has yet to be fully elucidated, but is likely to include the following: obstruction of CSF flow within the basal cisterns and/ or ventricles by clotted blood, diminished absorption at the arachnoid granulations, and inflammation. 5,9,20,30 Prior reports have demonstrated no significant benefit in the reduction of shunt-dependent hydrocephalus when performing microsurgical aneurysm treatment alone when compared with endovascular treatment. ${ }^{8,37}$ Since several of the factors involved in the pathogenesis of hydrocephalus are likely transient, we and others have hypothesized that creation of alternative CSF flow pathways through microsurgical fenestration of anatomical membranes containing CSF (e.g., the LT and the MoL) would help relieve obstruc- tions to CSF flow and prevent chronic shunt-dependent hydrocephalus in patients following aSAH. A number of groups have previously investigated whether fenestration of the LT alone reduces shunt-dependent hydrocephalus following aSAH with varied results. ${ }^{3,4,8,12-15,26,29,35}$ A more recent systematic review, however, failed to find any association between fenestration of the LT and reduced incidence of post-aSAH shunt-dependent hydrocephalus, casting doubts on the efficacy of this technique. ${ }^{14}$

Isolated fenestration of the LT fails to restore CSF flow from infratentorial to supratentorial cisternal compartments. Following SAH, the MoL often collects clotted blood and is thickened and inflamed. This creates loculation of CSF in the prepontine and interpeduncular cisterns that we informally refer to as the "fifth ventricle." It is common to have dramatic escape of CSF on opening of the MoL during subarachnoid dissection of the aneurysm, and Yaşargil has reported this as well in a large subset of patients. ${ }^{34}$ Given these observations, we hypothesized that tandem fenestration of the LT and MoL would restore communication between infratentorial and supratentorial CSF compartments and would open a clear CSF flow pathway to the arachnoid granulations over the cerebral convexity where CSF is reabsorbed. We systematically applied a tandem fenestration technique to the LT and MoL in all cases of ruptured aneurysms from January 2014 onward; the technique was used in patients undergoing a pterional or orbitozygomatic craniotomy for surgical clipping in whom there was sufficient surgical access to reach both anatomical structures. In the present article, we demonstrate a marked and statistically significant $82 \%$ reduction in shunt dependency with application of this tandem fenestration technique. The number needed to treat was 6.8 and was even lower in those at high risk for chronic hydrocephalus. There was also a statistically significant 27-day delay in those undergoing tandem fenestration who ultimately required a VP shunt, indicating CSF reabsorption that is improved relative to those not treated with tandem fenestration, but the effect was still insufficient to avoid shunting.

Consistent with a greater efficacy than isolated fenestration of the LT, tandem fenestration was associated with a shunt dependency rate of $3.2 \%$, which is lower than the reported $14 \%$ shunt dependency rate with isolated fenestration of the LT in pooled analysis..$^{14}$ In fact, this rate of shunt dependency is lower than rates of all reported case 
series of isolated LT fenestration with only one exception (reported range 2.3\%-25\%).,3,8,12,15,26,29,35 Two reports have previously evaluated the efficacy of tandem fenestration for reducing shunt dependency following aSAH with inconclusive results. ${ }^{2,28}$ In 1994, Sindou reported rates of shunt dependency of $15 \%$ in a group with a greater proportion of tandem fenestration. However, only $63 \%$ of the patients assigned to that series underwent tandem fenestration. ${ }^{28}$ In an article by Akyuz and Tuncer, rates of shunt dependency were similar between isolated and tandem fenestration, and the authors demonstrated a statistical trend toward reduction of shunt dependency with tandem fenestration than with fenestration of the LT alone (4\% vs $9.8 \%, \mathrm{LT}+\mathrm{MoL}$ vs LT alone, respectively), but the trend failed to reach statistical significance. ${ }^{2}$ Whether this discrepancy was the result of a smaller sample size $(n=145)$, restriction to anterior communicating artery aneurysms, and/or technical differences with microsurgical fenestration is not known.

To the best of our knowledge, the present study represents the largest case series $(n=663)$ to date reporting results with tandem microsurgical fenestration of the LT and MoL. It remains possible that our series actually underreported the effect size of this intervention. Consistent with prior reports, high Hunt and Hess grade, high Fisher grade, presence of intraventricular hemorrhage, and need for EVD placement predicted greater risk for shunt-dependent hydrocephalus following aSAH.,22,27,32 Likely reflective of changes in our regional referral patterns, patients treated at our center since 2014 have been, on average, older with more severe hemorrhage, as evidenced by higher Hunt and Hess and Fisher grades, as well as a greater proportion with intraventricular hemorrhage. Therefore, the cohort undergoing tandem microsurgical fenestration would be predicted to have higher rates of shunt dependency based on these factors alone and may partially mitigate the effect size of our intervention. ${ }^{1,22,27,32}$

Despite these promising findings, our study has limitations, and the results should be interpreted with caution. Our study is retrospective in design and without randomization, and therefore introduction of bias, including selection bias, cannot be excluded. Tandem microsurgical fenestration was systematically applied to all patients undergoing open surgical clip reconstruction of a ruptured aneurysm from January 2014 onward. Although no significant changes in the neurocritical care, EVD drainage, and/or clinical paradigms to assess need for a VP shunt occurred during the duration of this study, it remains possible that variations in these practices may confound the findings. Microsurgical fenestration of the LT and MoL requires removal of a large amount of subarachnoid blood from CSF cisterns, including the suprasellar, carotid, crural, and interpeduncular cisterns. Therefore, the relative contributions of greater evacuation of subarachnoid blood and alteration of CSF flow through tandem fenestration remain unclear. These findings are also based on a single institution and single surgeon's experience. These results should therefore be viewed as preliminary, and prospective, randomized multicenter studies are needed to confirm these results. Shunt-related morbidity remains high in modern neurosurgical practice and these studies are warranted..$^{10,22-24}$

\section{Conclusions}

Tandem fenestration of the LT and MoL during open aneurysm clipping and/or bypass is associated with reduced rates of shunt dependency following aSAH. These results suggest that this technique may be efficacious and should be applied to ruptured aneurysm of both the anterior and posterior circulations when the LT and $\mathrm{MoL}$ are surgically accessible. Future prospective randomized multicenter trials are warranted to confirm these promising preliminary results.

\section{Acknowledgments}

We would like to acknowledge Kenneth Probst for the illustration he provided for Fig. 1.

\section{References}

1. Adams H, Ban VS, Leinonen V, Aoun SG, Huttunen J, Saavalainen T, et al: Risk of shunting after aneurysmal subarachnoid hemorrhage: a collaborative study and initiation of a consortium. Stroke 47:2488-2496, 2016

2. Akyuz M, Tuncer R: The effects of fenestration of the interpeduncular cistern membrane arousted to the opening of lamina terminalis in patients with ruptured ACoA aneurysms: a prospective, comparative study. Acta Neurochir (Wien) 148:725-733, 2006

3. Andaluz N, Van Loveren HR, Keller JT, Zuccarello M: Anatomic and clinical study of the orbitopterional approach to anterior communicating artery aneurysms. Neurosurgery 52:1140-1149, 2003

4. Andaluz N, Zuccarello M: Fenestration of the lamina terminalis as a valuable adjunct in aneurysm surgery. Neurosurgery 55:1050-1059, 2004

5. Auer LM, Mokry M: Disturbed cerebrospinal fluid circulation after subarachnoid hemorrhage and acute aneurysm surgery. Neurosurgery 26:804-809, 1990

6. Connolly ES Jr, Rabinstein AA, Carhuapoma JR, Derdeyn CP, Dion J, Higashida RT, et al: Guidelines for the management of aneurysmal subarachnoid hemorrhage: a guideline for healthcare professionals from the American Heart Association/American Stroke Association. Stroke 43:1711-1737, 2012

7. de Rooij NK, Linn FH, van der Plas JA, Algra A, Rinkel GJ: Incidence of subarachnoid haemorrhage: a systematic review with emphasis on region, age, gender and time trends. J Neurol Neurosurg Psychiatry 78:1365-1372, 2007

8. Dehdashti AR, Rilliet B, Rufenacht DA, de Tribolet N: Shunt-dependent hydrocephalus after rupture of intracranial aneurysms: a prospective study of the influence of treatment modality. J Neurosurg 101:402-407, 2004

9. Dorai Z, Hynan LS, Kopitnik TA, Samson D: Factors related to hydrocephalus after aneurysmal subarachnoid hemorrhage. Neurosurgery 52:763-771, 2003

10. Greenberg JK, Washington CW, Guniganti R, Dacey RG Jr, Derdeyn CP, Zipfel GJ: Causes of 30-day readmission after aneurysmal subarachnoid hemorrhage. J Neurosurg 124:743-749, 2016

11. Hasan D, Vermeulen M, Wijdicks EF, Hijdra A, van Gijn $\mathrm{J}$ : Management problems in acute hydrocephalus after subarachnoid hemorrhage. Stroke 20:747-753, 1989

12. Kim JM, Jeon JY, Kim JH, Cheong JH, Bak KH, Kim CH, et al: Influence of lamina terminalis fenestration on the occurrence of the shunt-dependent hydrocephalus in anterior communicating artery aneurysmal subarachnoid hemorrhage. J Korean Med Sci 21:113-118, 2006

13. Komotar RJ, Hahn DK, Kim GH, Khandji J, Mocco J, Mayer 
SA, et al: The impact of microsurgical fenestration of the lamina terminalis on shunt-dependent hydrocephalus and vasospasm after aneurysmal subarachnoid hemorrhage. Neurosurgery 62:123-134, 2008

14. Komotar RJ, Hahn DK, Kim GH, Starke RM, Garrett MC, Merkow MB, et al: Efficacy of lamina terminalis fenestration in reducing shunt-dependent hydrocephalus following aneurysmal subarachnoid hemorrhage: a systematic review. Clinical article. J Neurosurg 111:147-154, 2009

15. Komotar RJ, Olivi A, Rigamonti D, Tamargo RJ: Microsurgical fenestration of the lamina terminalis reduces the incidence of shunt-dependent hydrocephalus after aneurysmal subarachnoid hemorrhage. Neurosurgery 51:1403-1413, 2002

16. Lai L, Morgan MK: Predictors of in-hospital shunt-dependent hydrocephalus following rupture of cerebral aneurysms. J Clin Neurosci 20:1134-1138, 2013

17. Lawton MT: Seven Aneurysms: Tenets and Techniques for Clipping. New York: Thieme, 2011

18. Lewis A, Taylor Kimberly W: Prediction of ventriculoperitoneal shunt placement based on type of failure during external ventricular drain wean. Clin Neurol Neurosurg 125:109113, 2014

19. Mackey J, Khoury JC, Alwell K, Moomaw CJ, Kissela BM, Flaherty ML, et al: Stable incidence but declining case-fatality rates of subarachnoid hemorrhage in a population. Neurology 87:2192-2197, 2016

20. Massicotte EM, Del Bigio MR: Human arachnoid villi response to subarachnoid hemorrhage: possible relationship to chronic hydrocephalus. J Neurosurg 91:80-84, 1999

21. Merkler AE, Ch'ang J, Parker WE, Murthy SB, Kamel H: The rate of complications after ventriculoperitoneal shunt surgery. World Neurosurg 98:654-658, 2017

22. Motiei-Langroudi R, Adeeb N, Foreman PM, Harrigan MR, Fisher WSR, Vyas NA, et al: Predictors of shunt insertion in aneurysmal subarachnoid hemorrhage. World Neurosurg 98:421-426, 2017

23. O'Kelly CJ, Kulkarni AV, Austin PC, Urbach D, Wallace MC: Shunt-dependent hydrocephalus after aneurysmal subarachnoid hemorrhage: incidence, predictors, and revision rates. Clinical article. J Neurosurg 111:1029-1035, 2009

24. Reddy GK: Ventriculoperitoneal shunt surgery and the incidence of shunt revision in adult patients with hemorrhage-related hydrocephalus. Clin Neurol Neurosurg 114:1211-1216, 2012

25. Rincon F, Gordon E, Starke RM, Buitrago MM, Fernandez A, Schmidt JM, et al: Predictors of long-term shunt-dependent hydrocephalus after aneurysmal subarachnoid hemorrhage. Clinical article. J Neurosurg 113:774-780, 2010

26. Schmieder K, Koch R, Lücke S, Harders A: Factors influencing shunt dependency after aneurysmal subarachnoid haemorrhage. Zentralbl Neurochir 60:133-140, 1999

27. Shigematsu H, Sorimachi T, Osada T, Aoki R, Srivatanakul K, Oda S, et al: Predictors of early vs late permanent shunt insertion after aneurysmal subarachnoid hemorrhage. Neurol Res 38:600-605, 2016

28. Sindou M: Favourable influence of opening the lamina termi- nalis and Lilliequist's membrane on the outcome of ruptured intracranial aneurysms. A study of 197 consecutive cases.

Acta Neurochir (Wien) 127:15-16, 1994

29. Tomasello F, d'Avella D, de Divitiis O: Does lamina terminalis fenestration reduce the incidence of chronic hydrocephalus after subarachnoid hemorrhage? Neurosurgery 45:827-832, 1999

30. van Gijn J, Hijdra A, Wijdicks EF, Vermeulen M, van Crevel $\mathrm{H}$ : Acute hydrocephalus after aneurysmal subarachnoid hemorrhage. J Neurosurg 63:355-362, 1985

31. Walcott BP, Iorgulescu JB, Stapleton CJ, Kamel H: Incidence, timing, and predictors of delayed shunting for hydrocephalus after aneurysmal subarachnoid hemorrhage. Neurocrit Care 23:54-58, 2015

32. Wilson CD, Safavi-Abbasi S, Sun H, Kalani MY, Zhao YD, Levitt MR, et al: Meta-analysis and systematic review of risk factors for shunt dependency after aneurysmal subarachnoid hemorrhage. J Neurosurg 126:586-595, 2017

33. Yamada S, Nakase H, Park YS, Nishimura F, Nakagawa I: Discriminant analysis prediction of the need for ventriculoperitoneal shunt after subarachnoid hemorrhage. J Stroke Cerebrovasc Dis 21:493-497, 2012

34. Yaşargil MG: Microneurosurgery. Stuttgart: Thieme, 1984, Vol 1

35. Yonekawa Y, Imhof HG, Ogata N, Bernays R, Kaku Y, Fandino J, et al: Aneurysm surgery in the acute stage: results of structured treatment. Neurol Med Chir (Tokyo) 38 Suppl: 45-49, 1998

36. Zacharia BE, Hickman ZL, Grobelny BT, DeRosa P, Kotchetkov I, Ducruet AF, et al: Epidemiology of aneurysmal subarachnoid hemorrhage. Neurosurg Clin N Am 21:221-233, 2010

37. Zaidi HA, Montoure A, Elhadi A, Nakaji P, McDougall CG, Albuquerque FC, et al: Long-term functional outcomes and predictors of shunt-dependent hydrocephalus after treatment of ruptured intracranial aneurysms in the BRAT trial: revisiting the clip vs coil debate. Neurosurgery 76:608-614, 2015

\section{Disclosures}

The authors report no conflict of interest concerning the materials or methods used in this study or the findings specified in this paper.

\section{Author Contributions}

Conception and design: Lawton, Winkler, Burkhardt. Acquisition of data: Winkler, Burkhardt, Rutledge, Rick, Partow, Yue, Birk, Bach, Raygor. Analysis and interpretation of data: Winkler, Burkhardt. Drafting the article: Winkler. Critically revising the article: Lawton, Winkler, Rutledge. Reviewed submitted version of manuscript: Winkler. Statistical analysis: Winkler, Yue.

\section{Correspondence}

Michael T. Lawton, Department of Neurological Surgery, Barrow Neurological Institute, 2901 N 3rd Ave., Phoenix, AZ 85013. email: michael.lawton@barrowbrainandspine.com. 